\title{
Synthesis of vertical graphene by microwave plasma enhanced chemical vapor deposition technique
}

\author{
Atul Bisht ${ }^{1}$, Sreekumar Chockalingam ${ }^{1}$, O. S. Panwar ${ }^{1}$, B. P. Singh ${ }^{2}$, Ajay Kesarwani ${ }^{1}$ and Jagdish Chand ${ }^{1}$ \\ ${ }^{1}$ Polymorphic Carbon Thin Films Group, Physics of Energy Harvesting, \\ ${ }^{2}$ Physics and Engineering of Carbon, Material Physics and Engineering Division, \\ CSIR-National Physical Laboratory, New Delhi -110012 (India) \\ E-mail: sreekuc@nplindia.org
}

\begin{abstract}
Vertical graphene was synthesized on nickel substrate using microwave plasma enhanced chemical vapor deposition technique by varying gas pressure from 5 to 30 Torr under various mixing ratios of argon, hydrogen and methane. The Raman spectra show two major fingerprints of graphene, 2D peak at $2700 \mathrm{~cm}^{-1}$ and $G$ peak $1580 \mathrm{~cm}^{-1}$. Scanning electron microscopy microstructure revealed flower like graphene structure which could find applications in gas sensing and field emission due to high surface-to-volume ratio.
\end{abstract}

Index Terms- Graphene, Microwave plasma enhanced chemical vapor deposition, Raman Spectroscopy, Scanning electron microscopy.

\section{INTRODUCTION}

Over the past few decades, synthesis and applicability of different form of carbon nanomaterial like carbon nanotubes, fullerenes, nanofiber, graphene etc have drawn much attention. Among these carbon based nanomaterial, graphenebased nanomaterials have recently drawn enormous attention owing to their remarkable mechanical, electronic, and optical properties [1]. Graphene, an isolated planar carbon sheet composed of a $\mathrm{sp}^{2}$ bonded honeycomb lattice is currently considered as one of the important materials due to its unique properties such as high electron mobility, high thermal conductivity and high mechanical strength [2]. Both horizontal and vertical oriented graphene have been reported in the literature $[3,4]$. The properties of graphene depend upon the number of layers presented and their stacking. Vertical graphene originate from stacking of several graphitic sheets along the $<001>$ direction and consists of domains coordinated with few layer graphene [5]. A notable feature of vertically oriented graphene is that the correlation between the two indices greatly deviates from that of ordinary graphitebased carbon materials. This correlation strongly suggests that vertical graphene are characterized by a high degree of graphitization in spite of the very small average size of the graphite regions [6]. Such deviation should give vertical graphene unique physical properties that might not be present in other graphite-based carbon materials. Recently, many researchers have shown interest in vertical oriented graphene due to their high surface-to-volume ratio compared to horizontal graphene. High surface-to-volume ratio is very much desirable for field emitters, gas sensors and biosensors applications [3].
Plasma-enhanced chemical vapor deposition (PECVD) techniques are commonly used to synthesize vertical graphene $[3,4]$. Compared to thermal chemical vapor deposition (TCVD), PECVD offers the advantages of low deposition temperature, higher growth rate and controlled microstructure which is critical for the semiconductor applications of graphene. Kim et al. [4] reported the synthesis of graphene down to $450{ }^{\circ} \mathrm{C}$ by using $2.45 \mathrm{GHz}$ microwave enhanced chemical vapor deposition (MW-PECVD) technique by using hydrogen and methane gas. MW-PECVD technique has advantage that the high power microwave produces high degree of ionization and plasma can be produced with different pressure ranging from few Torr to hundred Torr pressure.

In this present work, we describe the synthesis of vertical graphene using $2.45 \mathrm{GHz}$, MW-PECVD technique by varying gas mixing ratio of hydrogen, methane and argon. The as grown graphene film was characterized by scanning electron microscope (SEM) and Raman spectroscopy.

\section{EXPERIMENTAL DETAILS}

The vertical graphene was synthesized on nickel substrate by an indigenously developed MW-PECVD system which consists of $2.45 \mathrm{GHz}$ microwave generator capable of generating $1.2 \mathrm{~kW}$ power, isolator, stub tuner and water cooled cylindrical cavity. The chamber is separated with microwave generator unit and waveguide by a quartz plate. Sample heating was performed by a resistive heater controlled by proportional-integral-derivative (PID) controller. Nickel foil of size $10 \mathrm{~mm} \times 10 \mathrm{~mm}$ was used as catalyst substrate to grow vertical graphene. Substrates were treated with iso propenol and DI water before deposition of graphene. After inserting the substrate into deposition chamber, system was evacuated to $3 \times 10^{-7}$ Torr with the help of rotary and turbo molecular vacuum pump. Substrate temperature was increased to $650{ }^{\circ} \mathrm{C}$. Substrates were also cleaned at this temperature by hydrogen plasma upto 10 minute. Initially, pressure was increased to 1 Torr using Ar gas and then required deposition pressure was acquired by inserting hydrogen and methane gas mixture. Vertical graphene was grown at $700{ }^{\circ} \mathrm{C}$ by varying gas pressure from 5 to 30 Torr under various mixing ratios of argon, hydrogen and methane gas. The temperature was measured using a thermocouple positioned in the vicinity of the sample. In all the depositions run, time was fixed for 5 minute. Vertical graphene was characterized by scanning 
electron microscope (SEM) (EVO MA 10), atomic force microscope (AFM, model Veeco-V) for surface morphology. Raman spectra was taken by a Reninshaw spectrophoto meter (micro-Raman model inVia Reflex) with $514 \mathrm{~nm}$ laser excitation and notch filter cutting at $\sim 50 \mathrm{~cm}^{-1}$ at room temperature with $\sim 5 \mathrm{~mW}$ incident power.

\section{RESULTS \& DISCUSSIONS}

Raman spectroscopy is an important characterization technique to determine structural properties of carbon based nanostructure. Fig. 1 shows Raman spectra of vertical graphene deposited at three different pressures. It is evident from the figure that $\mathrm{D}$ peak and $\mathrm{G}$ peak dominate at all three pressures. D peak appeared at around $1350 \mathrm{~cm}^{-1}$ and $\mathrm{G}$ peak appeared at around $1590 \mathrm{~cm}^{-1}$. The former band corresponds to $\mathrm{D}$ band (after defected), activated by the disorder due to the finite crystallite size, vacancy, grain boundary etc, totally absent in HOPG while the latter band corresponds to $\mathrm{G}$ band (after graphite), indicating $E_{2 g}$ mode of graphite [7-9]. In the region of higher order Raman signal all samples presents two peak around $2690 \mathrm{~cm}^{-1}$ and $2930 \mathrm{~cm}^{-1}$ which appear in all graphitic based carbon films. Former peak is concerned to 2D peak and latter is concerned to the combination of $\mathrm{D}$ and $\mathrm{G}$

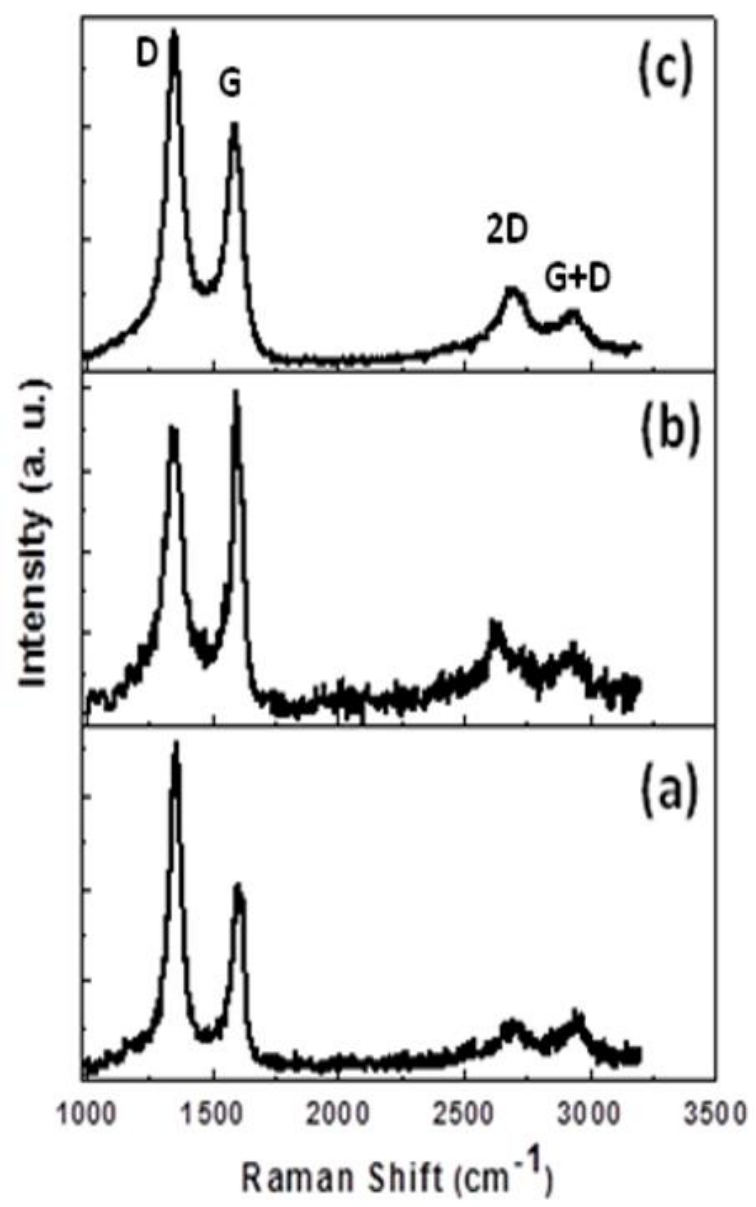

FIG.1. Raman spectra of vertical graphene deposited at different pressures of (a) 5 Torr, (b) 20 Torr and (c) 30 Torr.
(D+G) peak and also appear in Raman spectra of highly ordered pyrolytic graphite (HOPG). Full width at half maximum (FWHM) represents the quality of graphene layers. FWHM of 2D peak was found to vary from $116 \mathrm{~cm}^{-1}$ to 140 $\mathrm{cm}^{-1}$ depicting formation of few layer graphene. $\mathrm{D}+\mathrm{G}$ peak originate due to damage of graphene layer may be due to bombardment of high energy species present in plasma and microwave exposure. Table 1 summarizes the intensity ratio of $\mathrm{I}_{\mathrm{D}} / \mathrm{I}_{\mathrm{G}}, \mathrm{I}_{2 \mathrm{D}} / \mathrm{I}_{\mathrm{G}}$ and $\mathrm{FWHM}_{2 \mathrm{D}}$ and $\mathrm{FWHM}_{\mathrm{D}+\mathrm{G}}$ peaks evaluated from the Raman spectra of vertical graphene deposited at different pressures. The comparable intensity of $\mathrm{D}$ and $\mathrm{G}$ band may be due to the nanographite domains as revealed by the SEM image. $\mathrm{I}_{\mathrm{D}} / \mathrm{I}_{\mathrm{G}}$ represents degree of disorder present in carbon based nanostructure i.e., as $\mathrm{I}_{\mathrm{D}} / \mathrm{I}_{\mathrm{G}}$ ratio increase, the disorder in graphitic structure also increases. This ratio is minimum at pressure 20 Torr. High value of $\mathrm{FWHM}_{2 \mathrm{D}}$ reveals that the graphene domain comprising of multilayer graphene sheets. It is evident from the table that the value of $\mathrm{I}_{2 \mathrm{D}} / \mathrm{I}_{\mathrm{G}}$ is maximum and $\mathrm{I}_{\mathrm{D}} / \mathrm{I}_{\mathrm{G}}$ is minimum whereas $\mathrm{FWHM}_{2 \mathrm{D}}$ has minimum value accompanied with maximum value of $\mathrm{FWHM}_{\mathrm{D}+\mathrm{G}}$ at 20 Torr pressure which indicates that quality of vertical graphene is better at 20 Torr pressure. Peak intensity and FWHM of D, G, 2D and D+G band have been calculated

TABLE 1. Parameters of vertical graphene evaluated from Raman spectra at different pressures.

\begin{tabular}{clll}
\hline \hline Pressure/properties & 5 Torr & 20 Torr & 30 Torr \\
\hline $\mathrm{I}_{\mathrm{D}} / \mathrm{I}_{\mathrm{G}}$ & 1.68 & 0.88 & 1.42 \\
$\mathrm{I}_{2 \mathrm{D}} / \mathrm{I}_{\mathrm{G}}$ & 0.25 & 0.28 & 0.28 \\
$\mathrm{FWHM}_{2 \mathrm{D}}\left(\mathrm{cm}^{-1}\right)$ & 128.3 & 116.2 & 140.7 \\
$\mathrm{FWHM}_{\mathrm{D}+\mathrm{G}}\left(\mathrm{cm}^{-1}\right)$ & 107.5 & 153.2 & 109.2 \\
\hline \hline
\end{tabular}

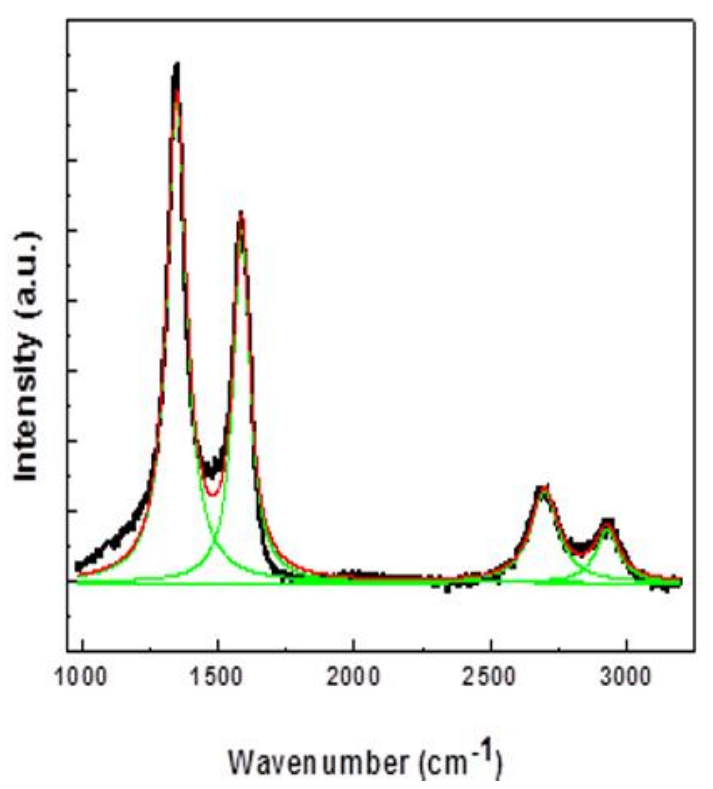

FIG. 2. Lorentzian fitting of Raman spectra of vertical graphene deposited at 20 Torr pressure. 

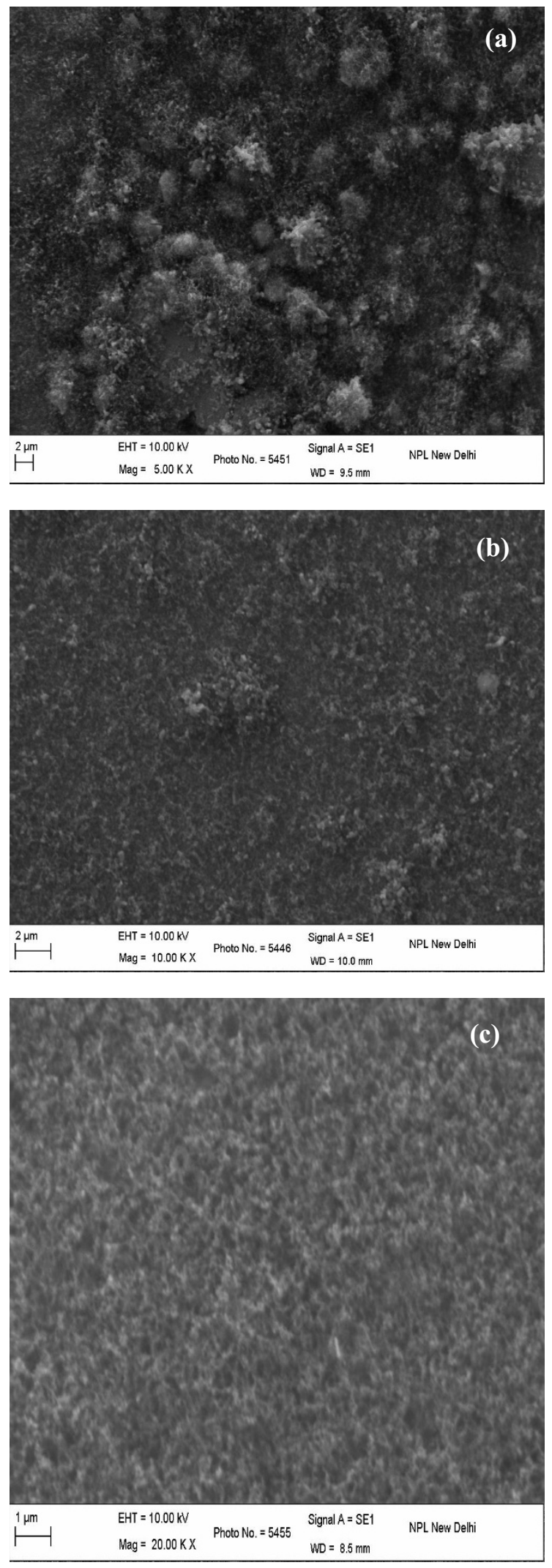

FIG. 3. Typical SEM micrographs of vertical graphene deposited at different pressures of (a) 5 Torr, (b) 20 Torr and (c) 30 Torr. by Lorentzian fitting of Raman spectra as shown in Fig. 2 . Thus, 20 Torr pressure is found to be optimum for obtaining better quality graphene. The $\mathrm{D}$ band is typically assigned to defects like grain boundary, vacancy, substitution by atom etc. Generally, high intense D band is the characteristic feature of these types of vertical graphene structure due to the presence of high number of edges which themselves act as defects. Fig. 3 shows the SEM micrographs of the vertical graphene deposited at different pressures. It is evident from the micrographs that there is formation of vertical graphene. Flower like structures are also present with high density at a pressure of 5 Torr and 20 Torr. These micrographs consist of small graphene domains attached in flower like structure. One can clearly see that with increasing pressure, the density of these graphene domains arranged in flower structure increases.

The electric field in the plasma promotes the growth at the edge of carbon nanosheet and induces vertical growth perpendicular to the substrate's surface [10]. Many growth models have been proposed for the formation of this type of structure in literature $[5,11]$. The nucleation of vertical graphene starts from the growth of small disordered graphitic sheets parallel to substrate. As deposition continues nanosheet formation with random direction occur due to continuous supply of necessary radical to grow by plasma assisted decomposition of precursor gas. Among these randomly oriented nanosheets, vertically oriented sheets continue to grow faster in vertical direction [12]. SEM micrographs clearly show uneven growth of flower like graphene in a matrix of small vertical sheet like structure. One can argue that the growth of flower like structure is mainly depending upon the nucleation time. Generally in the growth of graphene by chemical vapor deposition (CVD), the precursor gas decomposes due to the catalytic effect of substrate at high

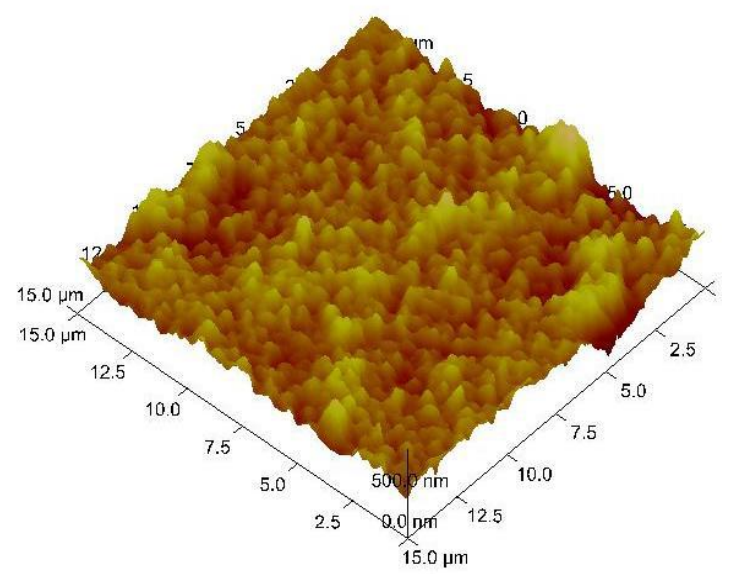

FIG. 4. Typical AFM micrograph of vertical graphene deposited at 20 Torr pressure.

temperature followed by cooling with high cooling rate. So, the carbon atoms dissolve into nickel segregated to surface to form graphene sheet $[13,14]$. This is not the mechanism of graphene growth in PECVD case as precursor decomposes by collision of high energy electron present in plasma and the building block are directly supplied by plasma to nucleate and grow the vertical structured graphene film. Fig. 4 represents 
the typical AFM micrograph of vertical graphene deposited at 20 Torr pressure. AFM micrograph reveals the vertical structure of uneven size graphene deposited at 20 Torr pressure. Vertical structure of uneven size of AFM micrograph corroborate with the structure appearing in SEM micrograph. Due to these vertical structures average roughness has high value upto $54.2 \mathrm{~nm}$. Further work, by varying the temperature and ratio of methane and hydrogen is in progress.

\section{CONCLUSIONS}

Vertical graphene is synthesized using MW-PECVD technique. Raman spectroscopy along with SEM and AFM studies confirmed the formation of graphitic nature of vertically oriented graphene sheets. Optimum pressure of deposition of vertical graphene in this study is found to be 20 Torr. These vertically oriented carbon sheets may find application in the area of field emission and gas sensors due to high aspect ratio and high surface area.

\section{ACKNOWLEDGEMENT}

The authors are grateful to Prof. R. C. Budhani, Director, CSIR-National Physical Laboratory, New Delhi (India), for his kind permission to publish this paper. They wish to thank Mr. A. K. Sood for providing the SEM micrographs, Mr. Sandeep Singh for providing AFM micrograph and R. K. Tripathi, for his help and useful discussion. Mr. Atul Bisht and Mr. Ajay Kumar Kesarwani are grateful to the University Grant Commission (UGC) and Council of Scientific Industrial Research (CSIR), Government of India, respectively, for financial assistance during the course of this work.

\section{REFERENCES}

[1] A. K. Geim. Science, 324, 1530 (2009).

[2] K. Novoselov, A. Geim, S. Morozov, D. Jiang, Y. Zhang, S. Dubonos, I. Grigorieva, A. Firsov, Science, 306, 666 (2004).

[3] Z. Bo, Y Yang, J. Chen, K. Yu, J. Yan, K. Cen, Nanoscale, 5, 5180, (2013).

[4] Y. Kim, W. Song, S. Y. Lee, C. Jeon, W. Jung, M. Kim, C. Y. Park, Appl. Phys. Lett., 98, 263106 (2011).

[5] K. Kobayashi, M. Tanimura, H. Nakai, A. Yoshimura, H. Yoshimura, K. Kojima and M. Tachibana, J. Appl. Phys., 101, 094306 (2007).

[6] Y. Wu, B. Yang, B. Zong, H. Sun, Z. Shen, and Y. Feng, J. Mater. Chem., 14, 469 (2004).

[7] R. J. Nemanich, S. A. Solin, Phys. Rev. B, 20, 392 (1979).

[8] S. Kurita, A. Yoshimura, H. Kawamoto, T. Uchida, K. Kojima, M. Tachibana, P. Molina Morales, H. Nakai, J. Appl. Phys., 97, 104320 (2005).

[9] Z. H. Ni, H. M. Fan, Y. P. Feng, Z. X. Shen, B. J. Yangand, Y. H. Wu, J. Chem. Phys., 124, 204703 (2006).

[10] M. Zhu, J. Wang, B. C. Holloway, R. A. Outlaw, X. Zhao, K. Hou, V. Shutthanandan D. M. Manos, Carbon, 45, 2229 (2007).

[11] I. Levchenko, K. Ostrikov, A. E. Rider, E. Tam, S. V. Vladimirov, S. Xu, Phys. Plasmas, 14, 063502 (2007).

[12] M. Hiramatsu, Y. Nihashi, H. Kondo, M. Hori, Jpn. J. Appl. Phys., 45, 5522 (2006).

[13] Q. Yu, J. Lian, S. Siriponglert, H. Li, Y. P. Chen, S. S. Pei, Appl. Phys. Lett., 93, 113103 (2008).

[14] L. Huang Q. H. Chang, G. L. Guo, Y. Liu, Y. Q. Xie, T. Wang, B. Ling, H. F. Yang, Carbon, 50, 551 (2012). 